\author{
Marquette University \\ e-Publications@Marquette
}

$10-2012$

\title{
Fabrication Process Comparison and Dynamics Evaluation of Electrothermal Actuators for a Prototype MEMS Safe and Arming Devices
}

\author{
Scott A. Ostrow II \\ Air Force Institute of Technology \\ Robert A. Lake \\ Air Force Institute of Technology \\ J. P. Lombardi III \\ Air Force Institute of Technology \\ Ronald A. Coutu Jr. \\ Marquette University, ronald.coutu@marquette.edu \\ Lavern A. Starman \\ Air Force Institute of Technology
}

Follow this and additional works at: https://epublications.marquette.edu/electric_fac

Part of the Computer Engineering Commons, and the Electrical and Computer Engineering Commons

\section{Recommended Citation}

Ostrow, Scott A. II; Lake, Robert A.; Lombardi, J. P. III; Coutu, Ronald A. Jr.; and Starman, Lavern A., "Fabrication Process Comparison and Dynamics Evaluation of Electrothermal Actuators for a Prototype MEMS Safe and Arming Devices" (2012). Electrical and Computer Engineering Faculty Research and Publications. 362.

https://epublications.marquette.edu/electric_fac/362 
Marquette University

\section{e-Publications@Marquette}

\section{Electrical and Computer Engineering Faculty Research and Publications/College of Engineering}

This paper is NOT THE PUBLISHED VERSION; but the author's final, peer-reviewed manuscript. The published version may be accessed by following the link in the citation below.

Experimental Mechanics, Vol. 52, No. 8, (October, 2012): 1229-1238. DOI. This article is (C) Springer and permission has been granted for this version to appear in $\underline{\mathrm{e}-}$ Publications@Marquette. Springer does not grant permission for this article to be further copied/distributed or hosted elsewhere without the express permission from Springer.

\section{Contents}

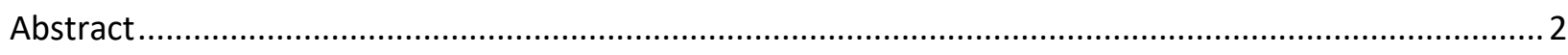

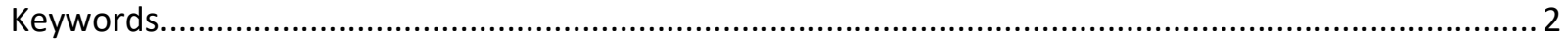

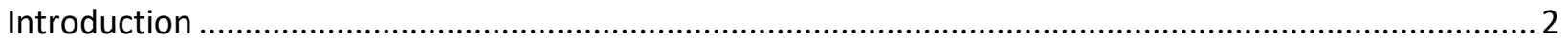

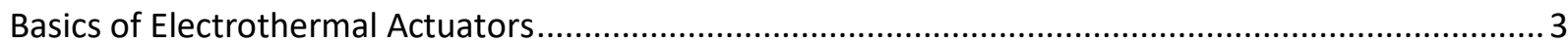

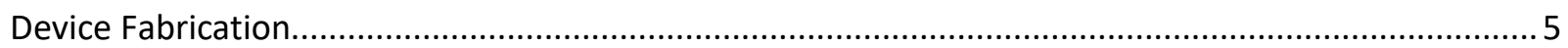

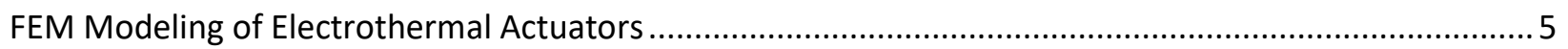

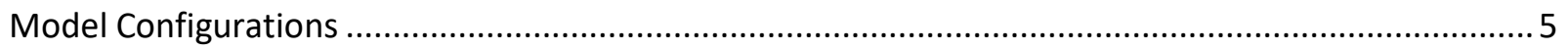

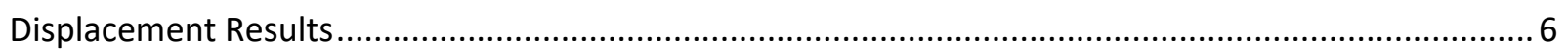

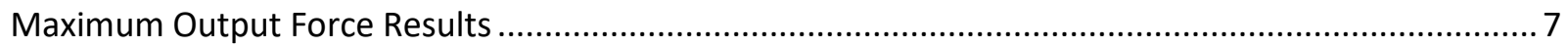

Dynamic Evaluation of Electrothermal Actuators Fabricated using SUMMiT and PolyMUMPs .................9

Integration of Electrothermal Actuators in a MEMS Safe and Arm Device ........................................... 11

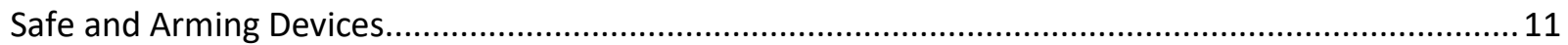

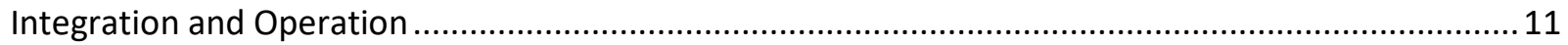

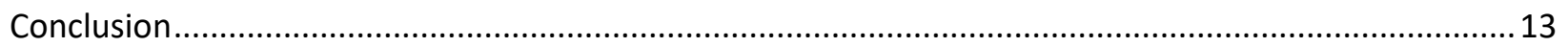

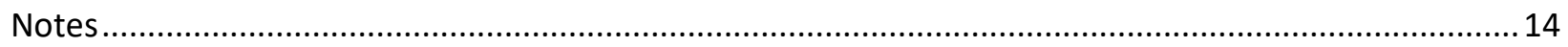

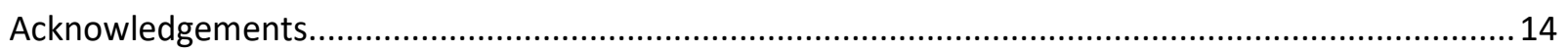

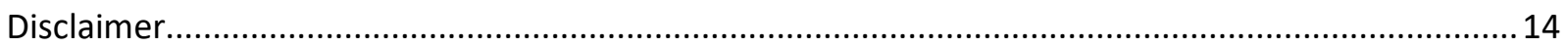




\section{Fabrication Process Comparison and Dynamics Evaluation of Electrothermal Actuators for a Prototype MEMS Safe and Arming Devices}

\section{S. A. Ostrow II}

Air Force Institute of Technology, $\mathrm{OH}$

R. A. Lake

Air Force Institute of Technology, $\mathrm{OH}$

J. P. Lombardi III

Air Force Institute of Technology, $\mathrm{OH}$

R. A. Coutu Jr.

Air Force Institute of Technology, $\mathrm{OH}$

L. A. Starman

Air Force Institute of Technology, $\mathrm{OH}$

\section{Abstract}

Electrothermal actuators fabricated using the Polysilicon Multi-User MEMS Process (PolyMUMPs) and the Sandia Ultra-Planar, Multi-Level MEMS Technology 5 (SUMMiT V) have been investigated for use in integrated microelectromechanical systems (MEMS) safe and arming devices. The fabricated electrothermal actuators have been dynamically tested to determine and compare the responses of devices from both processes. Furthermore, the integration of these devices into a safe and arming device were tested and investigated for each process. Initial results indicate that the SUMMiT devices provide the most optimum results based on consistency of operation and reliability.

Keywords

Electrothermal actuators; Microelectromechanical systems (MEMS); PolyMUMPs; SUMMiT; Safe and arm

\section{Introduction}

Electrothermal actuators have been utilized in a wide range of applications, such as drive motors for gear assemblies, self-assembly, and micro-optical applications., 1,2,3.4 They have been prototyped using fabrication processes like the Polysilicon Multi-User microelectromechanical systems (MEMS) Process 


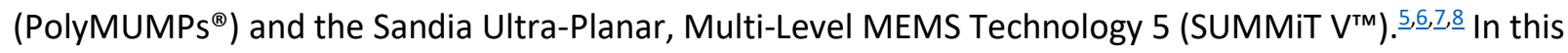
paper, electrothermal actuators and arrays were designed and fabricated in both processes and then tested and compared. Figure 1 shows an array of ten grouped thermal actuators fabricated in the PolyMUMPs process, and Fig. $\underline{2}$ shows a similar configuration fabricated in the SUMMiT process. The configurations were first modeled and analyzed using the finite element modeling (FEM) tool CoventorWare. This was followed by an examination of the dynamic response of the actuator arrays using a Zygo NewView 7300 white light interferometer modified with a dynamics test module. $\cdot$ Finally, a prototype MEMS-based safe and arming device integrated with electrothermal actuator arrays was investigated. $\underline{10}$

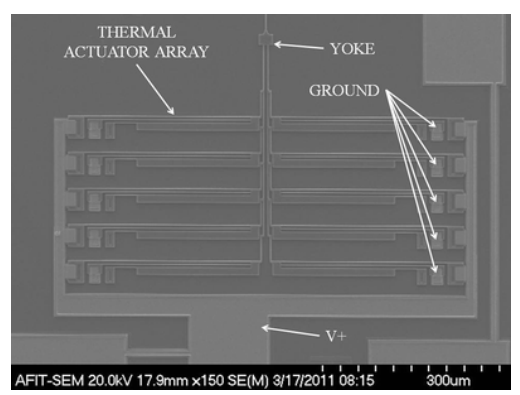

Fig. 1 SEM of an array of ten grouped thermal actuators fabricated in the PolyMUMPs fabrication process

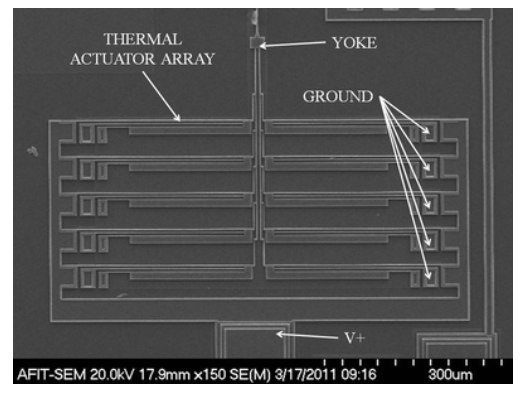

Fig. 2 SEM of an array of ten grouped thermal actuators fabricated in the SUMMiT fabrication process

\section{Basics of Electrothermal Actuators}

Electrothermal actuation takes advantage of the phenomena of thermal expansion of materials based on Joule heating generated by current flow. The property of any given material that dictates how the material will expand relative to a change in temperature is called the coefficient of thermal expansion (CTE). ${ }^{1}$ As an example, looking at a beam with initial length $L_{0}$, when the beam undergoes a temperature change, the new length of the beam, $L_{n e w}$, is given as:

$$
L_{\text {new }}=L_{0}+\propto_{L}\left[L_{0}\left(T_{\text {avg }}-T_{0}\right)\right](1)
$$

where $\alpha_{L}$ is the CTE, $T_{a v g}$ is the average temperature of the beam after the expansion, and $T_{0}$ is the initial temperature of the beam. ${ }^{2}$ This transduction from the application of a voltage (and the resulting current) to movement is rather complex and involves device resistance, material resistivity, energy dissipation, heat transfer, and thermal expansion.

Electrothermal actuators operate based on the asymmetrical thermal expansion of two arms made out of the same conductive material. For example, in laterally driven thermal actuators, as illustrated in Fig. $\underline{3}$, two beams (arms) are fabricated in the same material, have the same length, are connected at 
the ends, but have different cross-sectional areas. The wider cold arm has a much lower resistance than the narrower hot arm due to its higher cross sectional area, and the higher resistance of the hot arm results in a larger temperature increase localized in the hot arm relative to the cold arm due to a higher power dissipation. When current is passed through the device, the hot arm heats up faster and therefore has a larger thermal and linear expansion. This expansion causes the hot arm to bend towards the cold arm, and since the two arms are connected at the ends the entire device deflects in an arcing motion in the direction of the cold arm, as shown in Fig. $\underline{3(b))^{1,2}}$

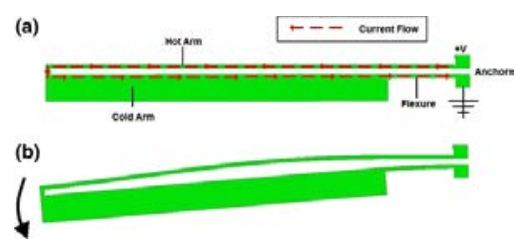

Fig. 3 Single lateral hot arm thermal actuator (a) at rest, (b) actuated

Where the energy is dissipated in the actuator is a direct result of the resistance of the device, which changes as a function of material thickness based on:

$$
R=p \frac{l}{A}(2)
$$

where $\rho$ is the resistivity of the material, $l$ the length of the structure, and $A$ the cross sectional area. The difference in thickness thus means a difference in resistivity in the two arms of the actuator, and this difference in resistance corresponds to a difference in the energy dissipated in different parts of the actuator by:

$$
P=I^{2} R(3)
$$

where $I$ is the current going through the actuator. This is referred to as Joule, or ohmic, heating. Using this basic principle, a variety of different configurations can be made to produce actuators that are unior bi- directional and actuate laterally or vertically. $, 4,4,5,6,7, \underline{8}$

Another configuration known as a double lateral hot arm actuator works on the same principles as the single hot arm actuator, as illustrated in Fig. 4 . The major difference is that instead of one narrow hot arm, there are two narrow hot arms in which the current is confined. Like the single hot arm thermal actuator, the two hot arms expand much more relative to that of the cold arm, which in this case undergoes minimal thermal expansion, causing the actuator to deflect in the direction of the cold arm. $\frac{6,11}{}$ The specific differences between these variations of electrothermal actuators will be discussed in a subsequent section.

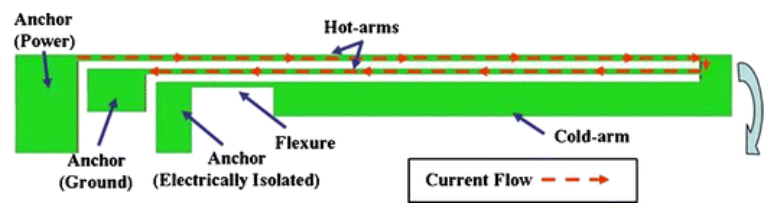

Fig. 4 Double lateral hot arm thermal actuator 


\section{Device Fabrication}

As mentioned above, the devices were fabricated using two different surface micromachining methods, PolyMUMPs and SUMMiT V. Both of these fabrication processes use similar thin film deposition methods and selective etching of polysilicon and silicon dioxide layers to create MEMS structures. PolyMUMPs consists of three polysilicon layers, two that are available as mechanical/structural layers and a third that serves as a fixed ground layer. The advantages of this process are that it is straightforward, has a rapid turn-around time of about two months, is relatively inexpensive, and allows for a certain degree of flexibility with its design rules. Disadvantages include a large minimum feature size and spacing of 2-4 $\mu \mathrm{m}$, non-uniformly doped device layers, a design limitation of two structural layers, and conformance in all the layers to the underlying topography, meaning the layers are not planar. $\underline{12}$

SUMMiT consists of five polysilicon layers, four that are available as mechanical/structural layers and a fifth that serves as ground layer. It offers several advantages over the PolyMUMPs process, such as tighter tolerances, smaller minimum feature sizes and spacing of 0.5-1 $\mu \mathrm{m}$, and two additional releasable structural layers, which allow for more complex designs. ${ }^{7}, \underline{9}$ In addition, the top two layers of this process are planarized by backfilling with silicon dioxide and then using chemical mechanical polishing to create a flat surface. This allows the top two structural layers to be planar, even if there is a rough underlying topography, providing a tremendous advantage when stacking devices on top of other structures, such as sliding plates and gears. Additional benefits include the in situ doping of the polysilicon layers resulting uniformly doped structural layers and minimal residual stress upon release. There are however some disadvantages of the SUMMiT process, such as, the turn-around time of 4 to 5 months for processing, and a price that is considerably more expensive than PolyMUMPs. Also, because of the added complexity of designs possible with the SUMMiT process, the layout and design requires considerably more effort and meticulous care, as there is little leniency when it comes to the design rules. $\underline{13}$

\section{FEM Modeling of Electrothermal Actuators}

\section{Model Configurations}

Multiple configurations of single and double hot arm thermal actuators, as well as multiple configurations of arrayed thermal actuators, were modeled and simulated using the MEMS finite element modeling software suite, CoventorWare. These models were "fabricated" within the software using the PolyMUMPs fabrication process provided by, CoventorWare. Three models of single hot arm actuators and three models of double hot arm actuators were analyzed. All the simulated devices had mechanical layers that were $3.5 \mu \mathrm{m}$ thick to mimic PolyMUMPs devices with stacked layers of Poly 1 and Poly2. All the cold arms were $20 \mu \mathrm{m}$ wide, all the hot arms were $3 \mu \mathrm{m}$ wide, and each flexure was $50 \mu \mathrm{m}$ long. The remaining variable parameters are summarized in Tables $\underline{1}$ and $\underline{2}$. In addition to these six models, four models consisting of thermal actuators all of the same size in groups of two, four, six and eight were analyzed. Figure $\underline{5}$ is an example of an array of four thermal actuators connected together with a coupling yoke. The characteristics of particular interest for this analysis were the displacement and maximum output force of the actuators. The relevant material properties used in the CoventorWare simulations are summarized in Table $\underline{3 .}^{14}$ Although polysilicon films with grain sizes on the order of $500 \mathrm{~nm}$ (i.e. PolyMUMPs) generally exhibit anisotropic material properties, the polysilicon layers used 
here were assumed to have isotropic material properties since the polysilicon film thickness was greater than $1 \mu \mathrm{m}$ and the actuator geometries were on the order of tens to hundreds of microns. $\underline{15}$

Additionally, residual stress, in the stacked mechanical layers, was assumed negligible and the layers were modeled as a stress free since the as fabricated devices were not curled or warped after release.

Table 1 Modeled length dimensions of single hot arm thermal actuators

\begin{tabular}{|l|l|l|l|}
\hline & Device A & Device B & Device C \\
\hline Cold Arm Length & $250 \mu \mathrm{m}$ & $300 \mu \mathrm{m}$ & $350 \mu \mathrm{m}$ \\
\hline Hot Arm Length & $300 \mu \mathrm{m}$ & $350 \mu \mathrm{m}$ & $400 \mu \mathrm{m}$ \\
\hline
\end{tabular}

Table 2 Modeled length dimensions of double hot arm thermal actuators

\begin{tabular}{|l|l|l|l|}
\hline & Device D & Device E & Device F \\
\hline Cold Arm Length & $250 \mu \mathrm{m}$ & $300 \mu \mathrm{m}$ & $350 \mu \mathrm{m}$ \\
\hline Inner Hot Arm Length & $313 \mu \mathrm{m}$ & $363 \mu \mathrm{m}$ & $413 \mu \mathrm{m}$ \\
\hline Outer Hot Arm Length & $346 \mu \mathrm{m}$ & $396 \mu \mathrm{m}$ & $446 \mu \mathrm{m}$ \\
\hline & & \\
\hline
\end{tabular}

Fig. 5 An illustration of an array of four thermal actuators connected together at their tips with a coupling yoke

Table 3 Polysilicon material properties used in the CoventorWare simulations ${ }^{14}$

\begin{tabular}{|l|l|}
\hline Material Property & Value \\
\hline Young's Modulus & $160 \mathrm{GPa}$ \\
\hline Poisson's Ratio & .22 \\
\hline Coefficient of Thermal Expansion & $4.7^{*} 10^{-6}(1 / \mathrm{K})$ \\
\hline Thermal Conductivity & $32(\mathrm{~W} / \mathrm{m} / \mathrm{K})$ \\
\hline
\end{tabular}

\section{Displacement Results}

For each of the models, a varying potential from zero to twelve volts was applied at the input terminals. In the case of the single hot arm actuators, the potential was applied between the end of the hot arm and the end of the cold arm, as shown in Fig. $3(\mathrm{a})$. For the double hot arm actuators, the potential was applied between the two hot arms, with the cold arm left electrically isolated, as shown in Fig. $\underline{4}$. The results indicate that as applied potential increases, the displacement of the actuators increases. It is also revealed that the longer the thermal actuator is, the further it deflects for the same applied power for both the single and double hot arm actuators, as illustrated in Fig. $\underline{6}$. A comparison of the displacement 
versus the applied voltage for the single and double hot arm actuators indicates that for the same applied potential, a single hot arm thermal actuator will deflect further than a double hot arm actuator with the same cold arm length. These results are deceiving at first glance, and while it is true that the single hot arm actuators deflect further for the same applied potential, more power is also dissipated by the single hot arm actuator due to the lower resistance encountered in the current path. When a comparison is made between the single and double hot arm actuators in terms of displacement versus dissipated power, it can be seen that a single hot arm actuator and double hot arm actuator with the same cold arm length deflect approximately the same distance for the same applied power. An analysis of the grouped thermal actuators indicates that when actuators are grouped together, their deflection is not affected by the number of actuators in the group, as shown in Fig. $\underline{7}$. This is a logical result in that all the actuators grouped together are the same size, and as a result would have the same deflection characteristics, and therefore not hinder or enhance each other.

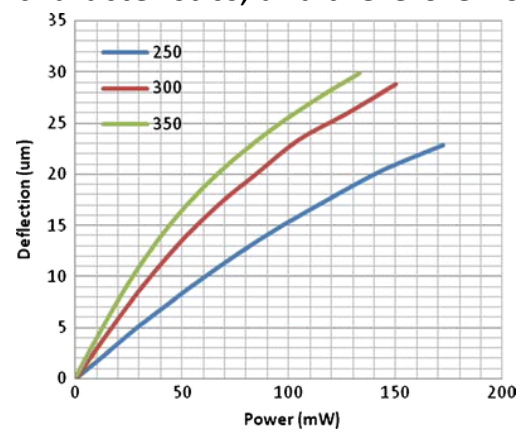

Fig. 6 Graph illustrating the displacement versus applied power of a 250, 300, and $350 \mu \mathrm{m}$ length thermal actuator. It can be seen that the longer thermal actuators deflect further for the same applied power

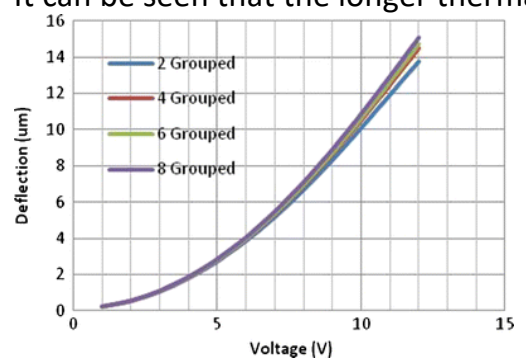

Fig. 7 Graph illustrating the grouped actuator deflection for arrays of two, four, six, and eight actuators. Note that the deflection remains very close to constant regardless of how many actuators are coupled together

\section{Maximum Output Force Results}

In order to determine the force exerted by the actuators, a special configuration is used as shown in Fig. $\underline{\text {. }}$. This configuration consists of a thermal actuator whose tip contacts a beam which is anchored at its other end. By knowing the parameters of this beam, treating it as a fixed-free beam, and then measuring how far the beam tip is displaced by the actuator, the maximum force generated, $F$, by the thermal actuator can be determined by:

$$
F=\frac{E t w^{3}}{4 l^{3}} x(4)
$$

where $E$ is the Young's modulus of the beam material, $t$ is the thickness of the beam, $w$ is the width of the beam, $l$ is the length of the beam, and $x$ is the distance that the beam is displaced. 


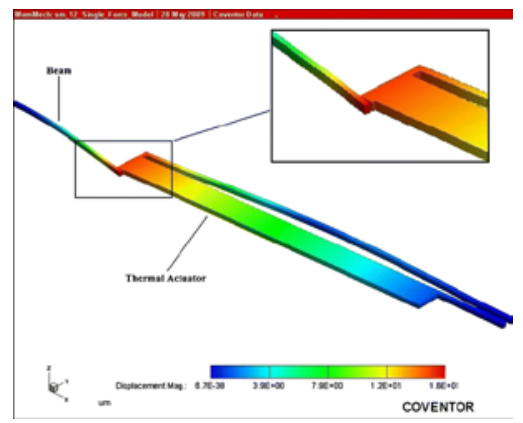

Fig. 8 Special configuration used to measure the maximum output force of a thermal actuator. The tip of the actuator contacts the tip of the beam and by measuring the lateral displacement of the beam, the applied force can be determined

The modeling results of the force characteristics of both the single and double hot arm thermal actuators demonstrate several interesting points that must be taken into consideration when designing devices with these actuators. For the single hot arm thermal actuators the results of the force analysis indicate that as the applied power to the actuator increases, the maximum output force generated by the actuator increases. More interestingly, there is a cross-over point for the three different lengths of actuators, as illustrated in Figure $\underline{9}$. At lower applied power, the longer actuators are capable of generating a higher output force, but as the applied power is increased a point is reached where the shorter actuators are capable of generating a higher output force. This can be attributed to the fact that as the actuators deflect they move in an arcing path. The longer ones will naturally arc more than the shorter actuators, resulting in more of the force being directed in another direction, and only a portion of its total output force being directed in the desired direction. These results reveal a trade-off that must be considered when designing with thermal actuators. In cases where larger deflections are desired, and force is not as important, the longer actuators would be ideal. In cases where larger forces are required, it is important to consider the input power that will be available and how much deflection is required, in order to choose which actuator is best suited for the design application.

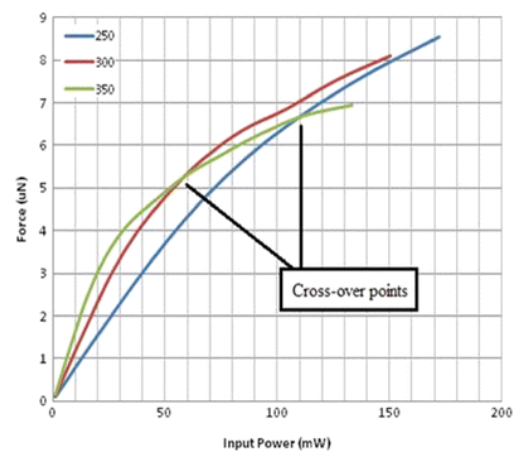

Fig. 9 Graph illustrating the FEM modeling results of the output force versus applied power of the single hot arm thermal actuators. Note the cross-over points at which the shorter actuators begin to generate a higher output force

Analysis of the double hot arm thermal actuators yields similar results. While the cross-over points are not as pronounced, it can be seen that at lower applied powers that the output forces are very similar, regardless of the actuator size. As the applied power increases, the output force of all the actuators increase and the shorter actuators are capable of generating higher output than the longer ones. A comparison of the force versus applied power characteristics of the single and double hot arm actuators 
indicates that the double hot arm thermal actuators posses much greater force generating capabilities. This clearly demonstrates that for situations in which a higher output force is required, the double hot arm thermal actuators are the more suitable choice.

Results of the force analysis for grouped thermal actuators reveal that there is a linear dependence of the output force on the number of thermal actuators that are grouped together, as shown in Figure 10. It is important to note that the increase of power consumption of these arrayed devices is directly proportional to the number of thermal actuators in the array. For example, an array with eight actuators will consume eight times the power of a single thermal actuator of the same configuration and dimensions. In situations where power consumption is limited this would be a critical design parameter. The results of modeling have provided important characteristics of different configurations of thermal actuators. With a better understanding of how they work and their performance characteristics, a choice as to which configurations are best suited for a particular application can more easily and accurately be made. In the case of the MEMS based safe and arming devices designed for this research, arrays of five and ten grouped double hot arm electrothermal actuators were utilized.

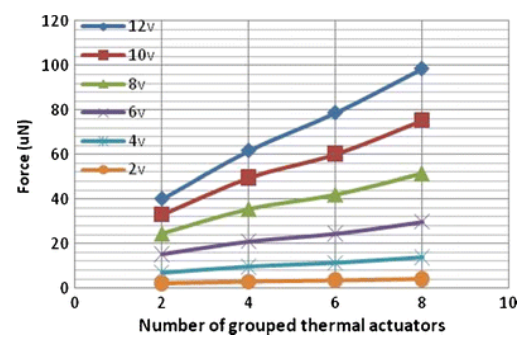

Fig. 10 Graph illustrating the linear dependence of the output force upon the number of thermal actuators within the array operating from 2 volts to 12 volts

\section{Dynamic Evaluation of Electrothermal Actuators Fabricated using SUMMiT and PolyMUMPs}

A dynamic analysis was undertaken because it allows for an assessment of the operation of the grouped thermal actuators at different operational frequencies. By comparing the relative response of similar devices fabricated in PolyMUMPs and SUMMiT, a determination of which fabrication process works best in terms of reliability, consistency, and response can be made. Arrays of five grouped thermal actuators and ten grouped thermal actuators fabricated in both PolyMUMPs and SUMMiT, were investigated. First, the actuators were tested on a probe station with a DC input, as well as a $500 \mathrm{~Hz}$ square wave input, to verify their operation. This was followed by dynamic measurements collected using a Zygo NewView 7300 across a range of frequencies, $500 \mathrm{~Hz}$ to $1.5 \mathrm{kHz}$ in $100 \mathrm{~Hz}$ increments. This range was utilized because the minimum frequency that can be utilized for the dynamic module of the Zygo is around $500 \mathrm{~Hz}$. The square wave input signal was approximately $10 V_{p p}$, with a $5 \mathrm{~V}$ offset to provide a $0-$ $10 \mathrm{~V}$ input, for the PolyMUMPs devices, and approximately $25 V_{p p}$, with a $12.5 \mathrm{~V}$ offset to provide a $0-$ $25 \mathrm{~V}$ input, for the SUMMiT devices. The dynamic measurements utilize stroboscopic illumination to take snap shots of the devices, in which the phase delay of the light source is varied between 00 and 360 o for each frequency and the motion of the device is captured based on the associated drive signal. The arrays of five and ten for each process were investigated individually, but it is important to note that the two arrays are attached to a common pawl system, which is key to the safe and arm application that 
will be discussed in the next section. Also, the applied voltage/power to the devices is tailored to the specific process, as highlighted above, and therefore will not be a direct comparison. For example, the SUMMiT devices require around 20-25 V to actuate, while the PolyMUMPs devices actuate around 10$15 \mathrm{~V}$. Applying 20-25 V to a PolyMUMPs device would typically result in device burnout and failure due to the resulting higher current.

Figures $\underline{11}$ and $\underline{12}$ highlight selected data on displacement versus frequency from example devices, which are consistent with what was observed throughout testing. The displacement values represent the maximum displacement that was observed at the given frequency. Key points to note are that the SUMMiT devices have a more consistent response, across the operational frequency range, then the PolyMUMPs devices and that the array of ten actuators has a more consistent response than the array of five actuators. Looking into the individual data points of the phase sweep at each frequency highlights the consistency of the SUMMiT devices, with the devices always in phase with the drive signal. This means that the maximum displacement is seen in conjunction with the maximum applied voltage level of the drive signal. In comparison, there are times with the PolyMUMPs devices where they are out of phase with the drive signal. This means that the maximum displacement is lagging behind the point of the maximum applied voltage level of the drive signal.

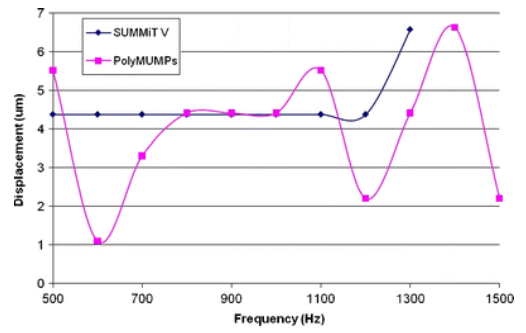

Fig. 11 Displacement versus frequency for five grouped thermal actuators

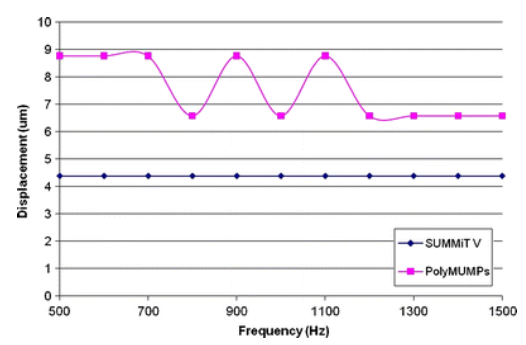

Fig. 12 Displacement versus frequency for ten grouped thermal actuators

The reasons for the differences between the similar devices are related to the different fabrication processes. The stacked SUMMiT MMPoly1-MMPoly2 layers, because they are thinner $(2.5 \mu \mathrm{m})$ and because they are doped in situ, tend to have a higher resistivity, and thus a higher thermal resistance than the stacked PolyMUMPs Poly1-Poly2 layers, which are thicker $(3.5 \mu \mathrm{m})$ and are doped via annealed phosphosilicate glass (PSG) masking layers. Additionally, the uniformly doped SUMMiT layers result in uniform current flow along the entire cross sectional area of the structure verses PolyMUMPs structures where the center of the mechanical layers are lightly doped (and more resistive) compared to the edges. $\underline{\underline{16}}$

This results in the SUMMiT devices needing higher voltages to actuate, and as a result are more immune to small variations in the drive voltage. On the other hand, the PolyMUMPs devices, because of their 
lower resistivity and thermal resistance, are more susceptible to these variations and thermal effects. There is a slight issue seen with the array of five SUMMiT devices starting around a frequency of $1.3 \mathrm{kHz}$. At this point the devices have a spike in actuation, as shown in Fig. 11 , and get stuck down to the substrate. Because of the higher tolerance of the SUMMiT devices as designed, they do not have enough leeway to deform/adapt to react to the higher frequencies, and as a result get stuck. Despite this issue at the higher frequencies, based on the results, the SUMMiT based designs appear to be the clear choice for the safe and arm application.

\section{Integration of Electrothermal Actuators in a MEMS Safe and Arm Device}

\section{Safe and Arming Devices}

The safe and arming device works on the principle of an interrupted explosive train. Simply put, a mechanical barrier exists between the initiator and the subsequent charge in the explosive train. The safe and arming device of the fuze is generally the largest component of a fuze and is mechanical in nature. As a result, it is an obvious target for miniaturization with MEMS components. Since the device is mechanical in nature, and requires the implementation of a movable barrier, some sort of actuation method is required in the design. Additionally, because of the nature of the device, mechanical safety locks must also be incorporated. According to the Department of Defense's Safety Criteria for Fuze Design, MIL-STD-1316E, safe and arming devices must contain two individual safety mechanisms. $\underline{17}^{17}$

\section{Integration and Operation}

Thermal actuators are used exclusively in the safe and arming device as it is currently designed and some considerations must be taken into account to effectively integrate these actuators. In the PolyMUMPs design, the moving barriers that interrupt the explosives are formed by rotating wheels, and in order for the device to be placed from a safe state into an armed state the outer edge of the wheel must be driven approximately $785 \mu \mathrm{m}$. This length is far beyond the maximum displacement capability of any electrothermal actuator. In order to convert the relatively small displacement of the thermal actuators into a larger usable displacement, a configuration known as a pawl is incorporated. The way in which this works is that two banks of actuators are driven perpendicularly to one another, $90^{\circ}$ out of phase. The first actuator array forces a drive head into contact with the object to be moved, as shown in Fig. 13(a). The second array then actuates, pushing the object in the same direction, as shown in Fig. 13(b). Once this movement has occurred, the first actuator array disengages, breaking the contact between the drive head and the moving object, as shown in Fig. 13(c). Finally, the second actuator array is allowed to return to its original position, as shown in Fig. 13(d). This process is repeated rapidly until the barrier has been positioned into an armed mode. Figure $\underline{14}$ shows a fabricated pawl mechanism for this application.

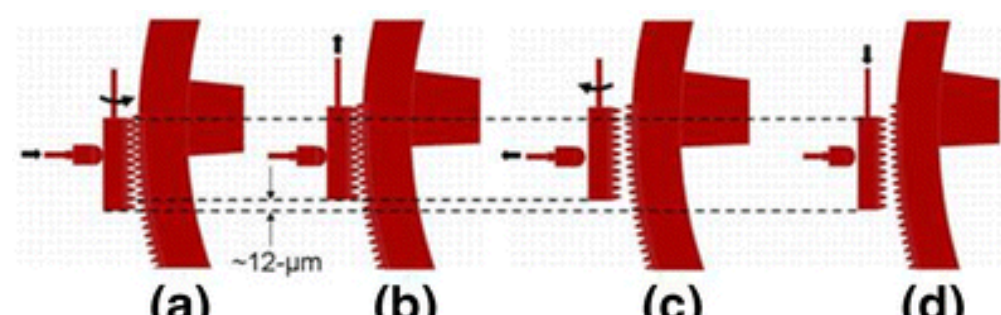
(a)
(b)
(c)
(d) 
Fig. 13 Pawl Operation: (a) Drive head is forced into contact with the wheel, (b) The drive head is pulled by the second array of actuators, (c) The drive head is disengaged from the wheel, (d) The drive head is returned to its original position

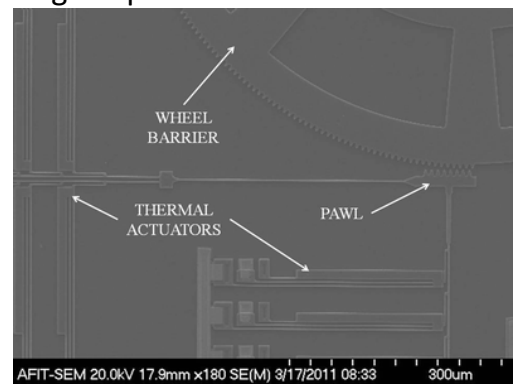

Fig. 14 SEM of pawl mechanism incorporated to rotate the wheel barrier of a MEMS based safe and arming device fabricated in the PolyMUMPs process

The time to open the wheel barrier can easily be predicted. The radius of the wheel is approximately $500 \mu \mathrm{m}$, which results in a distance to be traveled at the outer radius of the wheel to rotate one quarter turn to be placed in the armed mode. The distance between the teeth on the wheel is approximately $10 \mu \mathrm{m}$. Because the thermal actuators can produce a reliable displacement of 10 to $15 \mu \mathrm{m}$, it can be assumed that only one tooth is engaged per cycle, giving the wheel $10 \mu \mathrm{m}$ of travel per cycle. The opening time of the wheel can thus be predicted by the following relationship:

$$
T_{\text {open }}=\frac{78.5}{f_{\text {eff }}}(5)
$$

where $T_{\text {open }}$ is the time for the wheel to travel one quarter turn and $f_{\text {eff }}$ is the effective frequency of the drive signal in hertz. Because the drive signal is two pulse trains $90^{\circ}$ out of phase, as illustrated by Fig. 15, the frequency at which the wheel is driven is not the same frequency that is applied to each individual thermal actuator array. The effective period of the composite drive signal is from the beginning of the leading signal to the end of the lagging signal, essentially adding one quarter of an individual period to the effective period. The effective frequency, therefore, is given as:

$$
f_{\text {eff }}=\frac{f}{1.25}(6)
$$

where $f$ is the frequency of the individual drive signals.

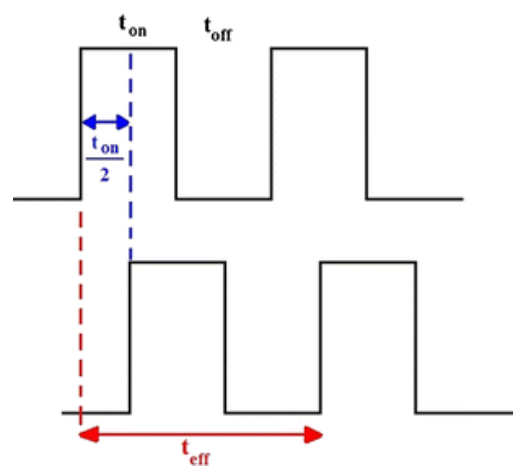

Fig. 15 Timing diagram of the thermal actuator drive signal 
As an example, Table $\underline{4}$ provides a comparison between predicted opening times and measured opening times for the PolyMUMPs design. The discrepancies between the predicted and measured opening times can be attributed to the fact that the pawl head may intermittently engage two teeth instead of one per cycle, causing it to open faster than predicted. In the case of the SUMMiT design, the wheel barrier is replaced with movable plates moved by a drive wheel that is controlled by a electrothermal actuator driven pawl mechanism, as shown in Fig. 16. Additional thermal actuators have been incorporated in both designs to serve as safety mechanisms as required by MIL-STD-1316E. These safeties simply prevent the barriers from moving when they are engaged. By applying a potential to these actuators, they disengage, allowing the barriers to move. The safeties of the PolyMUMPs device fully actuate the required $10 \mu \mathrm{m}$ when a potential of $12 \mathrm{~V}$ is applied, while the device fabricated in SUMMiT require a potential of $25 \mathrm{~V}$ in order to actuate the same distance. As mentioned previously, this is due to the fact that as polysilicon is deposited in the SUMMiT V process, it is doped in situ, causing it to have a higher resistance than polysilicon that is deposited using the PolyMUMPs fabrication process. Furthermore, testing of the complete system for the designs showed reliable operation up to around $250 \mathrm{~Hz}$, while at higher frequencies slipping of the teeth and wheel stalling was observed.

Table 4 Comparison between predicted and measured opening times

\begin{tabular}{|l|l|l|}
\hline Frequency (Hz) (Individual) & Predicted Opening Time (s) & Measured Opening Time (s) \\
\hline 2.5 & 39.25 & 37.5 \\
\hline 25 & 3.925 & 3.60 \\
\hline 250 & 0.3925 & 0.325 \\
\hline
\end{tabular}

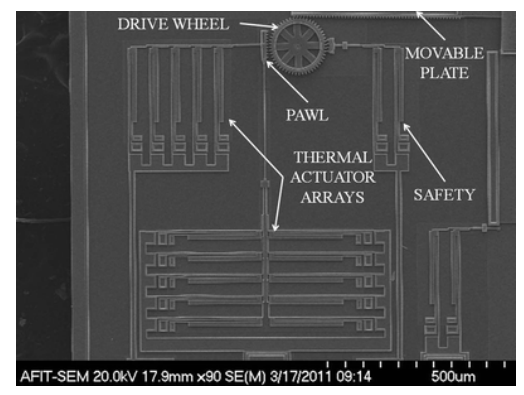

Fig. 16 SEM of a MEMS-based safe and arming device fabricated in the SUMMiT process

\section{Conclusion}

A fabrication process comparison and dynamics evaluation of electrothermal actuators for use in a prototype MEMS-based safe and arm devices were investigated. Based on dynamic testing results of arrays of thermal actuators fabricated in both the PolyMUMPs and SUMMiT V fabrication processes, it was shown that devices fabricated in the SUMMiT V process provided a more consistent and reliability response. These results also highlight the need for further dynamic testing of electrothermal actuator arrays as well as testing integrated safe and arm devices fabricated using PolyMUMPs and SUMMiT V. Planned future research includes additional dynamics testing to investigate vertical, out of plane 
deviations, due to higher order mechanical modes, as well as an investigation of polysilicon microstructure changes (e.g. grain size and boundary) due to current flow in electrothermal acutators.

\section{Notes}

\section{Acknowledgements}

Special thanks to Mr. Rick Patton for his assistance in troubleshooting the testing of devices for this research. Thanks also go to Captain Derrick Langley and Major Nathan Glauvitz for their insights and guidance.

\section{Disclaimer}

The views expressed in this paper are those of the authors and do not reflect the official policy or position of the United States Air Force, Department of Defense, or the U.S. Government.

\section{References}

1. Liu C (2005) Foundations of MEMS. Prentice Hall, Upper Saddle River, New Jersey

2. Kovacs GTA (1998) Micromachined transducers sourcebook. The McGraw Hill Company, Boston

3. Comtois JH, Bright VB (1997) Applications for surface-micromachined polysilicon thermal actuators and arrays. Sensor Actuator A 58:19-25

4. Yan D, Khajepour A, Mansour R (2004) Design and modeling of a MEMS bidirectional vertical thermal actuator. IOP, J Micromech Microeng 14:841-850

5. Comtois JH, Michalicek MA, Barron CC (1998) Electrothermal actuators fabricated in four-level planarized surface micromachined polycrystalline silicon. Sensor Actuator A 70:23-31

6. Kolesar E et al (2002) Single- and double-hot-arm asymmetrical polysilicon micromachined electrothermal actuators applied to realize a microengine. Thin Solid Films 420-421:530-538

7. Kolesar E et al (2000) In-plane tip deflection and force achieved with asymmetrical polysilicon electrothermal microactuators. Thin Solid Films 377-378:719-726

8. Kolesar E et al (2004) Design and performance of an electrothermal MEMS microengine capable of bi-directional motion. Thin Solid Films 447-448:481-488

9. Grigg $D$ et al (2004) Static and dynamic characterization of MEMS and MOEMS devices using optical interference microscopy. Proc SPIE 5455-55:1-7

10. Lake RA, Starman LA, Coutu RA Jr (2010) "Electrothermal actuators for integrated MEMS safe and arming devices," Proceedings of the Society for Experimental Mechanics, MEMS and Nanotechnology Conference 2:215-222

11. Yan D, Khajepour A, Mansour R (2003) Modeling of two-hot-arm horizontal thermal actuator. IOP, J Micromech Microeng 13:312-322

12. Carter J, Cowen A, Hardy B, Mahadevan R, Stonefield M, Wilcenski S. PolyMUMPs design handbook revision 11.0 .

13. Sandia National Laboratories, SUMMiT V - Five Level Surface Micromachining Technology Design Manual, April 2008.

14. CoventorWare (2010) MEMS design and analysis tutorials, Vol. 2, www.coventor.com.

15. Cho SW, Chasiotis I (2007) Elastic properties and representative volume element of polycrystalline silicon for MEMS. J Exp Mech 47:37-49 
16. Starman LA, Coutu RA Jr (2009) "Residual stress monitoring of post-processed MEMS fixed-fixed beams," Proceedings of the Society for Experimental Mechanics, MEMS and Nanotechnology Conference 2:769-778

17. MIL-STD-1316E, "Safety Criteria for Fuze Design." Department of Defense Design Criteria Standard, Jul 1998. 